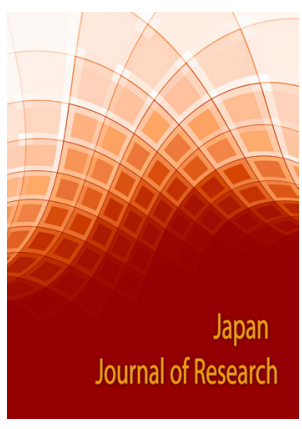

Correspondence

Ryan Nolan

Department of Dental Research, Novus Research, 730 E 950 S apt. A232, Orem, UT 84097, USA

Tel: 1-779-206-9804

Email: Ryannolandmd@gmail.com

- Received Date: 01 Jan 2020;

- Accepted Date: 12 Jan 2020;

- Publication Date: 17 Jan 2020.

\title{
Cytotoxicity of novel plant-based silver nanoparticles on fibroblasts for use in dental and medical applications
}

\author{
Craig Callister, Matt Callister, Michael Nolan and Ryan Nolan \\ Department of Dental Research, Novus Research, USA
}

\begin{abstract}
Background: The aim of this study was to evaluate the cytotoxicity of novel plant-based silver nanoparticles (AgNPs) on mammalian fibroblast cells.

Materials and methods: An in vitro prospective, double blind study model was used. A range of 5-17.5 ppm of the novel AgNPs were used in a neutral red uptake cytotoxicity assay. All test samples were compared to a negative and positive control to assess validity.

Results: The novel AgNPs showed non-cytotoxicity at all concentrations tested. All tested values were significant compared to the positive controls which showed cytotoxicity $(<70 \%$ cell viability) via one way anova $(p<0.05)$.

Conclusion: The novel AgNPs show promise for use in preventative applications for Dentistry and Medicine. The novel AgNPs could be used as a non-toxic alternative to Chlorhexidine and

Cetylpyridinium Chloride.
\end{abstract}

\section{Introduction}

(c) 2020 Science Excel. This is an openaccess article distributed under the terms of the Creative Commons Attribution 4.0 International license.
Nanotechnology has garnered much attention in recent years due to its multiple applications in various industries including medicine and dentistry. Silver nanoparticles have seen wide publication due to their extensive antimicrobial action and broad range of use. One of the major concerns for silver nanoparticles has been their inconsistent nature surrounding toxicity. Silver nanoparticle toxicity has been attributed to many factors including the methods of fabrication, as well as the capping agents used. Ultimately, the stability of these nanoparticles in biological media has been determined by many to be size and capping agent dependent [1]. It was therefore the authors' goal to test various concentrations of novel plant-based silver nanoparticles, which show greater promise for use as dental and medical preventative agents. By stabilizing and capping silver nanoparticles with novel plant-based compounds, the toxicity of these particles could be severely reduced. Synthesis methods with less toxicity will ultimately open doors for broader acceptability of nanotechnology in the medical and dental fields.

With the advent of new technology, there are always concerns regarding cytotoxicity and toxicity for mammalian species. Studies involving nanotechnology have rendered multiple useful publications and systemic reviews. However, one of the main concerns surrounding nanotechnology to this day is safety for human use. Despite many innovations in the field, many silver nanoparticles still suffer from instability, especially in high ionic strength environments and biological media [2].
It has been cited that this is primarily due to the methods of production, including harsh bases, additives, and stabilizers [3]. It should also be noted that if instability is present, especially in biological media, in vitro experiments may show the results of destabilized nanoparticles. Unfortunately, although many silver nanoparticles have shown promise for antimicrobial or chemotherapeutic use, their stability and toxicity profiles do not always meet the standard for use in vivo. Therefore, new methods are required to improve upon these shortcomings in order to develop a more stable and biocompatible alternative for use in the medical and dental fields.

New research has suggested that instead of using harsh bases and additives, natural plant compounds can be used [4]. Although this idea is not new, it has shown great promise for reduced cytotoxicity and increased biocompatibility. One of the major problems with plant compounds in the past has been their lacking ability to completely convert silver salts into silver nanoparticles [4]. Although some silver nanoparticles can be formed, the majority of the salt input remains in solution, resulting in an incomplete or undesirable reaction. Due to this lack of reducing power, plant compounds have been utilized mostly as secondary stabilizing agents. Unfortunately, plant compounds have not seen much success as single use alternatives to chemically modified and reduced nanoparticles. It can be noted that unless plant compounds are capable of containing high reduction potential in addition to providing particle stabilization, they will fail to produce a complete conversion into size consistent silver nanoparticles [4]. 
As research has improved and more plant compounds have become available, their usage for creating complete and stable conversions to nanoparticles has become possible. This is because certain plant compounds have seen increased purification from manufacturers and have become more commercially available. With increased commercial availability, these compounds can be manufactured at grades which make them more ideal reducing agents, without losing their biocompatibility. This has opened up entirely new possibilities for silver nanoparticle fabrication which previously relied on harsh chemical methods for production.

One of the largest downsides from previous generations of silver nanoparticles has been their lack of biocompatibility [5]. This has been evident from testing in vitro in various biological media. Many authors have speculated that this is due to lacking stability, size profiles, and capping agent selection $[5,6]$. One of the most popular methods for making silver nanoparticles with sodium citrate reduction has resulted in nanoparticles that can destabilize, especially when exposed to salts or various biological media [6]. As a result, their citrate barriers degrade, leading to large effluxes of silver ions in addition to agglomeration. Paired to this, methods which have used DMSO, Sodium borohydride and other harsh bases have encountered similar problems due to low stability in biological media as well as toxicity issues from the reducing agent, and resulting efflux of ions [7]. Many authors have suggested that the size of the nanoparticles is to blame, but recent work suggests that size profiles do not always match toxicity results [8]. Therefore, the reducing agent, stability of the particles (agglomeration \& ion release), and the capping agent selection are likely the main cause of toxicity concerns via instability in various media [9]. By utilizing plant-based compounds these issues can be severely reduced. One of the major benefits of the traditional chemical method for producing silver nanoparticles is a complete conversion from silver salt to silver nanoparticles. This method also offers a highly consistent particle size. In contrast, plant-based compounds have faced criticism due to their poor reduction potential and as a result poor conversion rates. Despite this, researchers have always been hopeful to find plant compounds with high conversion rates due to their lower toxicity profiles compared to their chemical counterparts.

Due to improvements in technology, many purified plant compounds can now be secured and used to create silver nanoparticles. Many of these plant compounds show little to no toxicity and can be made with a carbon-free footprint. This allows for the nanoparticle seeding process to occur more rapidly, increasing conversion via bio-reduction. This has led to the capability to produce completely converted silver nanoparticles which are not only non-cytotoxic, but still maintain their biocompatibility and size consistency compared to their chemically produced counterparts [10].

\section{Materials and Methods}

\section{Characterization of nanoparticles}

Silver nanoparticles, $<5-10 \mathrm{~nm}>$ in size capped with a novel plant-based compound were obtained from Nanocomposix, CA. Characterization was performed by Nanocomposix, CA using good laboratory practices. The size of the nanoparticles was measured using JEOL 1010 (TEM) via counts of 300 to confirm particle size distribution. Thermo Fisher Xseries II (ICP-MS) was used to measure the conversion of the nanoparticles from silver salts into fully formed nanoparticles. A UV-VIS analysis was done to confirm the wavelength and conversion of the particles to even size distributions at predictable wavelengths for silver nanoparticles utilizing an Agilent 8453 spectrophotometer. Additionally, nanoparticles were tested for Zeta potential and hydrodynamic diameter using Malvern Zetasizer Nano ZS. Nanoparticle concentrations were diluted from the original base solution after being verified for concentration via ICP-MS. Each test was performed in triplicate to thoroughly analyze the particles. Figure 1 illustrates the TEM \& UV-VIS results.
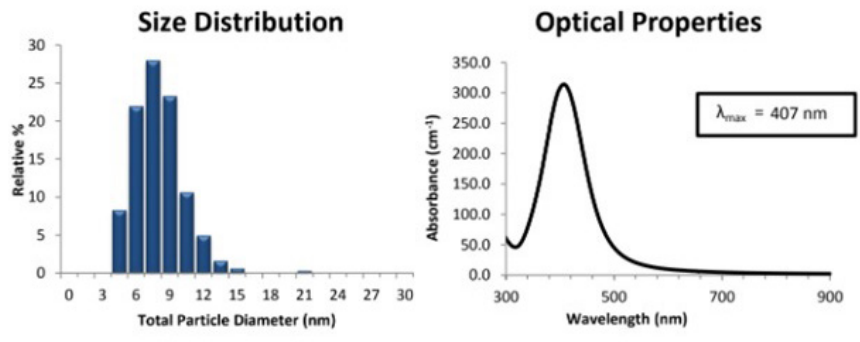

Figure 1. TEM \& UV-VIS analysis of Silver Nanoparticles (AgNPs)

\section{Cell culture}

The Cell culture selected was Balb/ c 3t3 mouse fibroblast cells. This is due to their comparability to Human fibroblast cells and in common relation to Human gingival and PDL cells surrounding the ligature which supports tooth structure to bone. Cells were obtained from ATCC and prepared using a seeding concentration of 1 E105 cells $/ \mathrm{mL}$ and incubated at $37+/-10 \mathrm{C}$ with $+/-1 \% \mathrm{CO}_{2}$. The cells were prepared in DMEM Maintenance growth media for Balb/ c 3t3 cells (DMEM supplemented with 10\% NBCS, Hepes, and Penicillin-Streptomycin). DMEM contained L-Glutamine already added. 100uL of prepared cell suspension was added to each well of a 96 well plate with the exception of the outer rows and columns of wells, equating to $1 \mathrm{E} 104$ cells per well. Prior to use, cells were microscopically verified to consist of a subconfluent monolayer with healthy morphology. Cells were tested for mycoplasma and determined to be mycoplasma free.

\section{Experimental conditions}

Cell cultures were tested for cytotoxicity using the well-established ISO compliant neutral red (NR) uptake cytotoxicity assay. The assay measures viability of cells via lysosome activity to uptake neutral red stain. The neutral red uptake is measured photometrically on a plate reader and the number of viable cells correlates with the color intensity measured (absorbance). The Balb/ c 3t3 plates were prepared and incubated for 22 to 24 hours. DMEM 5\% extraction media was added to the vehicle control, negative control, and Zinc Dibutyldithiocarbamate (ZDBC) positive control according to the extraction ratio and incubated at $37+/-1^{\circ} \mathrm{C}$ for $24+/-2$ hours with continuous agitation. The positive control (SLS) stock was prepared at $10 \mathrm{mg} / \mathrm{mL}$ and diluted to specified concentrations $\quad(0.1 \mathrm{mg} / \mathrm{mL}, \quad 0.08 \mathrm{mg} / \mathrm{mL}, \quad 0.06 \mathrm{mg} / \mathrm{mL}, \quad 0.05 \mathrm{mg} / \mathrm{mL}$, $0.04 \mathrm{mg} / \mathrm{mL}$ ). Only the lowest four dilutions were used for testing purposes. The positive control was diluted, but the negative and vehicle control were not diluted. $100 \mathrm{uL}$ of each dilution was added to six wells of a plate and the vehicle control was added to a total of 12 wells on each plate. The plates were incubated at $37+/-1^{\circ} \mathrm{C}$ with $5+/-1 \% \mathrm{CO}_{2}$ and $85+/-15 \%$ humidity for $24+/-2$ hours. After incubation all media were removed and the plate was washed with $150 \mathrm{uL}$ of PBS. Once the wash was removed, $100 \mathrm{uL}$ of NR medium was added and the plate was incubated for 3 hours $+/-10$ minutes at $37+/-1^{\circ} \mathrm{C}$ with $5+/-1 \% \mathrm{CO}_{2}$ and $85+/-15 \%$ humidity. The NR medium was discarded, and the cells were washed with $150 \mathrm{uL}$ of PBS. The wash was removed and $150 \mathrm{uL}$ of desorbing fixative (EtOH/acetic acid solution) was added. The plate was gently tapped and placed on an orbital shaker and rapidly shaken for 10 minutes. The NR detection was detected using a microplate reader at $540 \mathrm{~nm}$. All nanoparticle test samples were blanked prior to reading in order to mitigate error in the final measurement. The positive controls were $0.25 \%$ Zinc Dibutyldithiocarbamate (ZDBC), and Sodium lauryl sulfate (SLS). The negative control was High density polyethylene (HDPE). The test articles were composed of silver nanoparticles (5$17.5 \mathrm{ppm}$ ) diluted from a $0.3 \%$ base solution. 


\section{Cytotoxicity}

The cell monolayer was inspected and verified to have an approximate $50 \%$ confluency alongside verification of a healthy morphology. ZDBC positive control was diluted in DMEM 5\% NCS (100\%, 40\%, 20\% dilutions). The vehicle and negative controls were not diluted. The maintenance media from the plates was removed from the wells using a multichannel pipette or plate inversions. $100 \mathrm{uL}$ of each dilution was aseptically added to 6 wells with the 96 well plate. 100uL of DMEM $+5 \%$ NCS media was added to the blanks. The vehicle control consisted of 12 wells. The Plates were labeled appropriately, and the dishes were incubated at $37+/-1^{\circ} \mathrm{C}$ with $5+/-1 \% \mathrm{CO}_{2}$ and $85+/-15 \%$ humidity for $24+/-2$ hours. After incubation, the cell monolayer was inspected for signs of cell death. All media was then removed from the wells with a micropipette or inversion of the plate. 150uL of pre-warmed PBS was added to each well and then removed by quickly inverting the plate. $100 \mathrm{uL}$ of NR solution was added to all wells. Plates were incubated for $3+/-10$ minutes at $37+/-1^{\circ} \mathrm{C}$ with $5+/-1 \% \mathrm{CO}_{2}$ and $85+/-15 \%$ humidity. Following the 3 hours $+/-10$-minute incubation period, the NR solution was removed from all 96 wells and the wells were washed with $150 \mathrm{uL}$ of pre-warmed PBS. The PBS was subsequently removed. $150 \mathrm{uL}$ of EtOH/acetic acid desorption solution was added to each well. The plate was shaken rapidly for 10 minutes, placed in a microplate reader and evaluated at $540 \mathrm{~nm}$. The optical density of the 6 wells for each neat concentration and dilution was averaged and compared to the optical density average of all 12 vehicle control wells. Optical density was measured according to equation.

$\%$ Viability $=($ OD Average of sample/ OD average of VC) X 100

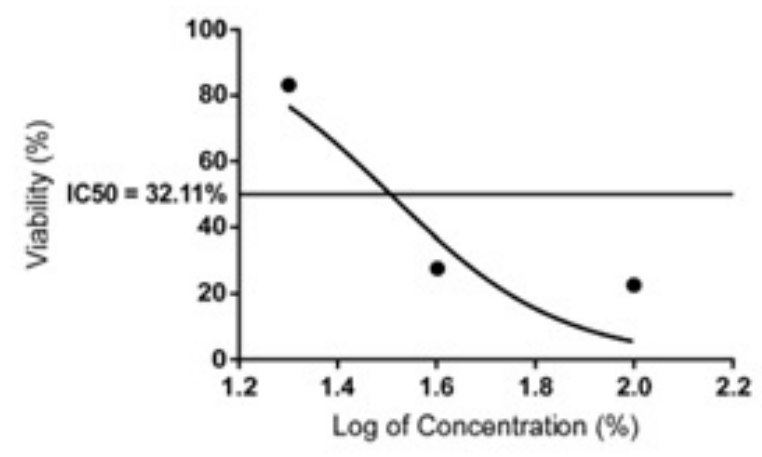

Figure 2. $Z D B C I C C_{50}$ positive control results

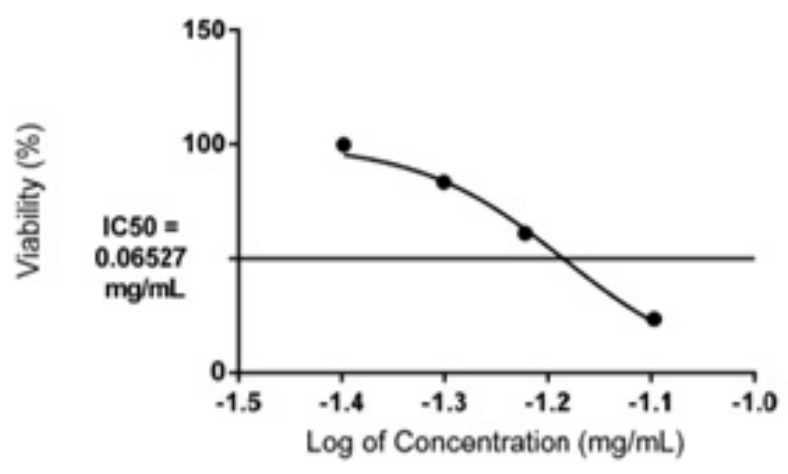

Figure 3. $\mathrm{SLSIC}_{50}$ positive control results
The $\mathrm{IC}_{50}$ value was calculated for the ZDBC positive control, SLS positive control and for the silver nanoparticle solutions. All articles were noted to be cytotoxic if they fell below 70\% cell viability. Graphpad prism and software for windows (San Diego, CA) was used for plot outputs of cell viability. The analysis was based off of the log values (inhibitor vs. normalized response-variable slope) equation built into the prism software. The values for the dilutions were converted to $\log _{10}$ form prior to analysis to conform to the requirements of the built in graphpad equation. Positive control results are shown below. Negative control results (HDPE) were $98.6 \%$ cell viability and are not shown below.

\section{Experimental setup - Nanoparticle analysis:}

UV-VIS \& ICP-MS analysis: Silver nanoparticles were fabricated and checked with UV-VIS and ICP-MS data. Verification that particles were completely formed was made confirming $99.99 \%$ conversion to nanoparticles. UV-VIS also confirmed shapes of peaks at the expected ranges of 400-420nm for silver nanoparticles. (Shown in Figure 1).

TEM analysis: Silver nanoparticles were tested via TEM to verify the average particle sizes. Silver nanoparticles were noted to be between 5-10nm, with an average particle size of $<7 \mathrm{~nm}>$. (Shown in Figure 1).

\section{Results}

\section{AgNP cytotoxicity}

As shown in Tables 1, 2 and 3, cytotoxicity results from the two positive controls showed cytotoxicity. The negative control and the AgNP test articles showed non-cytotoxicity. Silver nanoparticle test articles show that all concentrations (up to $17.5 \mathrm{ppm}$ ) were non-cytotoxic via one way anova $\mathrm{p}<0.05$ ( $>70 \%$ cell viability) when compared to positive vehicle controls. This lends credence to the idea that silver nanoparticles engineered using plant compounds are not only highly biocompatible, but are also slow to release ions from instability, especially in settings where exposure to multiple biological factors and high ionic strength are a concern. With the advent of highly pure plant compounds, it is clear that improvements upon previous generations of nanoparticles can be made. Experiments done show high validity profiles for inclusion into multiple biological settings, including medical imaging, chemotherapy, as well as oral biofilms.

\section{Results}

One of the major challenges in preventative oral care has been the lack of materials available for long-term use. This is apparent with quaternary ammonium compounds such as Chlorhexidine and Cetylpyridinium Chloride. These compounds have also been shown to have conflicting results subgingivally, eliciting challenges for practitioners treating periodontitis and gum disease [11]. The current FDA recommendations suggest to use Chlorhexidine for no longer than 2 weeks for gingivitis. Additionally, potential side effects include staining, taste changes, and calculus deposition. Ultimately, this can lead to reduced compliance with patient populations [12]. It has also been shown that Chlorhexidine and Cetylpyridinium Chloride can be toxic to fibroblasts, among other cell types $[13,14]$. Due to these vast limitations, it warrants exploration for using newer materials with less side effects, higher biocompatibility, and lower concentrations to prevent fibroblast death and reduced wound healing. It is evident from recent reports that Chlorhexidine is being used less post-surgically due to interruption of fibroblast, and osteoblast activity which may delay wound healing activity at surgical sites [15]. Recent studies on silver nanoparticles have shown that they are comparable to Chlorhexidine's antimicrobial action even at lower concentrations than their quaternary ammonium counterparts [16]. Certain studies have even shown that silver nanoparticles can reduce inflammation around surgical sites leading to reduced healing time [17]. With new technology, there exists 


\begin{tabular}{|c|c|c|c|c|c|c|c|c|c|c|}
\hline Sample ID & $\begin{array}{c}\log _{10} \text { for use in } \\
\text { Graphpad to } \\
\text { calculate IC10 }\end{array}$ & 1 & 2 & 3 & 4 & 5 & 6 & $\begin{array}{c}\text { Average } \\
\text { OD }\end{array}$ & $\begin{array}{l}\text { Standard } \\
\text { Deviation }\end{array}$ & $\begin{array}{c}\text { Viability } \\
(\%)\end{array}$ \\
\hline HDPE & NA & 0.336 & 0.557 & 0.452 & 0.449 & 0.564 & 0.495 & 0.476 & 0.084 & 98.6 \\
\hline ZDBC $100 \%$ & 2 & 0.084 & 0.153 & 0.074 & 0.099 & 0.092 & 0.151 & 0.109 & 0.034 & 22.6 \\
\hline ZDBC $40 \%$ & 1.602 & 0.175 & 0.139 & 0.101 & 0.09 & 0.118 & 0.181 & 0.134 & 0.038 & 27.7 \\
\hline ZDBC $20 \%$ & 1.301 & 0.349 & 0.312 & 0.301 & 0.595 & 0.382 & 0.473 & 0.402 & 0.113 & 83.2 \\
\hline SLS $0.08 \mathrm{mg} / \mathrm{ml}$ & -1.097 & 0.075 & 0.102 & 0.153 & 0.097 & 0.126 & 0.132 & 0.114 & 0.028 & 23.6 \\
\hline SLS $0.06 \mathrm{mg} / \mathrm{ml}$ & -1.222 & 0.209 & 0.445 & 0.271 & 0.411 & 0.201 & 0.233 & 0.295 & 0.106 & 61.1 \\
\hline SLS $0.05 \mathrm{mg} / \mathrm{ml}$ & -1.301 & 0.566 & 0.269 & 0.354 & 0.37 & 0.467 & 0.391 & 0.403 & 0.102 & 83.4 \\
\hline SLS $0.04 \mathrm{mg} / \mathrm{ml}$ & -1.398 & 0.495 & 0.494 & 0.614 & 0.463 & 0.434 & 0.391 & 0.482 & 0.076 & 99.8 \\
\hline Left Vehicle Control & NA & 0.403 & 0.527 & 0.542 & 0.385 & 0.625 & 0.568 & 0.508 & 0.095 & 105.2 \\
\hline Right Vehicle Control & NA & 0.305 & 0.638 & 0.501 & 0.449 & 0.494 & 0.361 & 0.458 & 0.117 & 94.8 \\
\hline Average VC & & \multicolumn{7}{|c|}{ Average OD of left and right vichle control } & 0.483 & \\
\hline
\end{tabular}

Table 1. Viability percentage of control article replicates

\begin{tabular}{|l|c|c|c|c|c|c|}
\hline Control Article & Concentration & $\begin{array}{c}\text { Cell Death } \\
\text { Observed }\end{array}$ & Average OD & \% Viability & IC $_{\mathbf{5 0}}$ & Interpretation \\
\hline Vehicle (left) & $\mathrm{NA}$ & None & 0.508 & 105.2 & NA & Non-Cytotoxic \\
\hline Vehicle (right) & $\mathrm{NA}$ & None & 0.458 & 94.8 & NA & Non-Cytotoxic \\
\hline Negative - HPDE & $100 \%$ & None & 0.476 & 98.6 & NA & Non-Cytotoxic \\
\hline & $100 \%$ & Complete & 0.109 & 22.6 & & \multirow{2}{*}{ Cytotoxic } \\
Postive - ZDBC & $40 \%$ & Complete & 0.134 & 27.7 & & \\
& $20 \%$ & Incomplete & 0.402 & 83.2 & & \\
& $0.08 \mathrm{mg} / \mathrm{ml}$ & Complete & 0.114 & 23.6 & & \multirow{2}{*}{ Cytotoxic } \\
& $0.06 \mathrm{mg} / \mathrm{ml}$ & Incomplete & 0.295 & $8.06527 \mathrm{mg} / \mathrm{ml}$ & \\
\hline
\end{tabular}

Table 2. Cytoxicity of control articles

\begin{tabular}{|c|c|c|c|c|c|c|c|}
\hline $\begin{array}{c}\text { Control } \\
\text { Article }\end{array}$ & $\begin{array}{c}\text { Concentration } \\
(\mathrm{ppm})\end{array}$ & $\begin{array}{l}\text { Cell Death } \\
\text { Observed }\end{array}$ & Average OD & $\%$ Viability & $\mathrm{IC}_{50}$ & $\begin{array}{l}\text { Standard } \\
\text { Deviation }\end{array}$ & Interpretation \\
\hline \multirow{4}{*}{$\begin{array}{l}\text { Nanosilver } \\
\text { (Set 1) }\end{array}$} & 17.5 & None & 0.504 & 109.6 & \multirow{4}{*}{ NA } & 0.118 & \multirow{4}{*}{ Non-Cytotoxic } \\
\hline & 15 & None & 0.504 & 109.6 & & 0.088 & \\
\hline & 12.5 & None & 0.528 & 114.8 & & 0.128 & \\
\hline & 10 & None & 0.444 & 96.5 & & 0.04 & \\
\hline \multirow{4}{*}{$\begin{array}{l}\text { Nanosilver } \\
\text { (Set 2) }\end{array}$} & 15 & None & 0.355 & 77.2 & \multirow{4}{*}{ NA } & 0.183 & \multirow{4}{*}{ Non-Cytotoxic } \\
\hline & 12.5 & None & 0.626 & 136.1 & & 0.168 & \\
\hline & 10 & None & 0.595 & 129.3 & & 0.207 & \\
\hline & 7.5 & None & 0.468 & 101.7 & & 0.092 & \\
\hline \multirow{4}{*}{$\begin{array}{l}\text { Nanosilver } \\
\text { (Set 3) }\end{array}$} & 12.5 & None & 0.668 & 138.9 & \multirow{4}{*}{ NA } & 0.112 & \multirow{4}{*}{ Non-Cytotoxic } \\
\hline & 10 & None & 0.575 & 119.5 & & 0.075 & \\
\hline & 7.5 & None & 0.562 & 116.8 & & 0.069 & \\
\hline & 5 & None & 0.553 & 115 & & 0.082 & \\
\hline
\end{tabular}

Table 3. Cytoxicity of AgNP test articles 
potential to incorporate new materials into existing regimens to help prevent disease on a cost-effective scale while reducing unnecessary exposure to multiple agents. As demonstrated in this study, fibroblasts were not affected at the concentrations chosen, showing that plantbased silver nanoparticles have the potential to replace chlorhexidine over time.

The cytotoxicity profiles for silver nanoparticles using a novel plantbased compound are ideal for use in dental and medical applications. One of the major problems with the physical and chemical methods used to produce silver nanoparticles is their genuine lack of protection in addition to biocompatibility and toxicity concerns. It is clear that these novel compounds have improved upon previous generations of nanoparticles and are feasible for extensive use in wound healing, control of microorganisms, among many other uses. One of the major facets of concern which has been blamed for cytotoxicity has been ion release over time. However, with newer biological based reduction methods, this may be mitigated and reduced. It is becoming clear that nanotechnology is advancing and has the potential to replace existing antimicrobials which suffer from many significant drawbacks.

\section{Conclusion}

Inclusion of nanoparticles into new materials has opened up doors for broad span application in multiple fields, including dentistry. Silver nanoparticles in particular have been of great interest due to their excellent antimicrobial action [18], use in caries prevention [19], and high activity against periodontal disease [20]. Although previous generations of silver nanoparticles suffered from toxicity concerns, it is becoming clear that highly stable and purified plant compounds can now be utilized for improving these materials downsides. All concentrations of silver nanoparticles tested showed non-cytotoxicity to mammalian fibroblasts. With this in mind, more broad span applications for nanotechnology in dental and medical preventatives can be made, alleviating the need for formerly more toxic profiles such as Cetylpyridinium Chloride and Chlorhexidine Gluconate.

\section{Conflict of interest statement}

This paper is thanks to the contributions and discussions of the authors on many occasions. We are thankful all of the authors contributions. Additional thanks goto APS Laboratories for cytotoxicity testing, and Nanocomposix for analysis of particles. Funding for this study was provided by Novus Research.

\section{Acknowledgements}

All Authors acknowledge ownership in Novus Research, and the patent submissions for the novel plant-based nanoparticles.

\section{References}

1. Niska K, Knap N, Kędzia A, et al. Capping agent-dependent toxicity and antimicrobial activity of silver nanoparticles: An in vitro study. concerns about potential application in dental practice. Int J Med Sci. 2016;13(10):772-782.

2. Yang $Y, X u S, X u G$, et al. Effects of ionic strength on physicochemical properties and toxicity of silver nanoparticles. Sci Total Environ. 2019;647:1088-1096.

3. Stensberg MC, Wei $Q$, Mclamore ES, et al. Toxicological studies on silver nanoparticles: challenges and opportunities in assessment, monitoring and imaging. 2011.

4. Billacura MP, Mimbesa HS. Leaf extract mediated green synthesis of silver nanoparticles from widely available wedelia trilobata: synthesis, partial characterization and antimicrobial property analysis. In: BMJ; 2015:bmjopen2015-forum2015abstracts.

5. Vasanth SB, Kurian GA. Toxicity evaluation of silver nanoparticles synthesized by chemical and green route in different experimental models. Artif cells, nanomedicine, Biotechnol. 2017;45(8):1721-1727.

6. Moore TL, Rodriguez-Lorenzo L, Hirsch V, et al. Nanoparticle colloidal stability in cell culture media and impact on cellular interactions. Chem Soc Rev. 2015;44(17):6287-6305.

7. Liao C, Li Y, Tjong SC. Molecular sciences bactericidal and cytotoxic properties of silver nanoparticles.

8. Huk A, Izak-Nau E, Reidy B, et al. Is the toxic potential of nanosilver dependent on its size? Part Fibre Toxicol. 2014;11(1).

9. McShan D, Ray PC, Yu H. Molecular toxicity mechanism of nanosilver. J Food Drug Anal. 2014;22(1):116-127.

10. Hembram KC, Kumar R, Kandha L, et al. Therapeutic prospective of plant-induced silver nanoparticles: application as antimicrobial and anticancer agent. Artif Cells, Nanomedicine Biotechnol. 2018;46(sup3):S38-S51.

11. Cosyn J, Wyn I. A Systematic review on the effects of the chlorhexidine chip when used as an adjunct to scaling and root planing in the treatment of chronic periodontitis. J Periodontol. 2006;77(2):257-264.

12. Vishnu Prasanna SG, Lakshmanan R. Characteristics, uses and side effects of chlorhexidine-a review. IOSR J Dent Med Sci e-ISSN. 2016;15(6):57-59.

13. Liu JX, Werner J, Kirsch T, et al. Cytotoxicity evaluation of chlorhexidine gluconate on human fibroblasts, myoblasts, and osteoblasts. J Bone Jt Infect. 2018;3(4):165172.

14. Fromm-Dornieden C, Rembe JD, Schäfer N, et al. Cetylpyridinium chloride and miramistin as antiseptic substances in chronic wound management - Prospects and limitations. J Med Microbiol. 2015;64(4):407-414.

15. Wyganowska-Swiatkowska M, Kotwicka M, Urbaniak P, et al. Clinical implications of the growth-suppressive effects of chlorhexidine at low and high concentrations on human gingival fibroblasts and changes in morphology. Int J Mol Med. 2016;37(6):1594-1600

16. Luckie RAM, Casatañares RL, Schougall R, Reyes SCG, Mendieta VS. Antibacterial effect of silver nanoparticles versus chlorhexidine against Streptococcus mutans and Lactobacillus casei. In: Silver Nanoparticles - Fabrication, Characterization and Applications. InTech; 2018.

17. Konop M, Damps T, Misicka A, Rudnicka L. Certain aspects of silver and silver nanoparticles in wound care: A minireview. J Nanomater. 2016;2016.

18. Qais FA, Shafiq A, Khan HM, et al. Antibacterial effect of silver nanoparticles synthesized using Murraya koenigii (L.) against multidrug-resistant pathogens. Bioinorg Chem Appl. 2019;2019.

19. Espinosa-Cristóbal LF, López-Ruiz N, Cabada-Tarín D, et al. Antiadherence and antimicrobial properties of silver nanoparticles against streptococcus mutans on brackets and wires used for orthodontic treatments. J Nanomater. 2018;2018.

20. Shawky H, Basha SM, Batouti G, et al. Evaluation of clinical and antimicrobial efficacy of silver nanoparticles and tetracycline films in the treatment of periodontal pockets. IOSR J Dent Med Sci Ver I. 2015;14(7):2279-2861. 